\title{
Comparison of targeted next-generation sequencing and Sanger sequencing for the detection of PIK3CA mutations in breast cancer
}

Ruza Arsenic ${ }^{*}$, Denise Treue, Annika Lehmann, Michael Hummel, Manfred Dietel, Carsten Denkert and Jan Budczies

\begin{abstract}
Background: Phosphatidylinositol-4,5-bisphosphate 3-kinase, catalytic subunit alpha, PIK3CA, is one of the most frequently mutated genes in breast cancer, and the mutation status of PIK3CA has clinical relevance related to response to therapy.

The aim of our study was to investigate the mutation status of PIK3CA gene and to evaluate the concordance between NGS and SGS for the most important hotspot regions in exon 9 and 20, to investigate additional hotspots outside of these exons using NGS, and to correlate the PIK3CA mutation status with the clinicopathological characteristics of the cohort.

Methods: In the current study, next-generation sequencing (NGS) and Sanger Sequencing (SGS) was used for the mutational analysis of PIK3CA in 186 breast carcinomas.

Results: Altogether, 64 tumors had PIK3CA mutations, 55 of these mutations occurred in exons 9 and 20. Out of these 55 mutations, 52 could also be detected by Sanger sequencing resulting in a concordance of $98.4 \%$ between the two sequencing methods. The three mutations missed by SGS had low variant frequencies below $10 \%$. Additionally, $4.8 \%$ of the tumors had mutations in exons $1,4,7$, and 13 of PIK3CA that were not detected by SGS. PIK3CA mutation status was significantly associated with hormone receptor-positivity, HER2-negativity, tumor grade, and lymph node involvement. However, there was no statistically significant association between the PIK3CA mutation status and overall survival.

Conclusions: Based on our study, NGS is recommended as follows: 1) for correctly assessing the mutation status of PIK3CA in breast cancer, especially for cases with low tumor content, 2) for the detection of subclonal mutations, and 3) for simultaneous mutation detection in multiple exons.
\end{abstract}

Keywords: Next-generation sequencing, Breast cancer, Sanger sequencing, PIK3CA

\section{Background}

Historically, Sanger sequencing (SGS) has been the gold standard for detecting DNA mutations. However, SGS has limitations due to its restricted sensitivity and its inability to perform parallel investigation of multiple targets. Furthermore, somatic cancer mutations can be difficult to detect using SGS without performing microdissections because tumors are heterogeneous and often mixed with normal tissue. Recent progress in massive

* Correspondence: ruza.arsenic@charite.de

Institute of Pathology, Charité University Hospital Berlin, Berlin, Germany parallel sequencing, termed next-generation sequencing (NGS), has increased the speed and efficiency of mutation testing in molecular pathology [1-9]. NGS allows for the detection of a broad spectrum of mutations, including single nucleotide substitutions, small insertions and deletions, large genomic duplications and deletions, and rare variations [9].

Targeted NGS, which involves the targeted enrichment of a set of DNA regions, is used for the parallel sequencing of amplicons derived from multiplex polymerase chain reaction (PCR) or other amplicon-based 
enrichment approaches, such as hybridization capture. When the amplicon size is kept small (e.g., <175 bp) in the design of the sequencing panel, NGS is also applicable to formalin-fixed tissue samples. Moreover, targeted NGS is more cost efficient than SGS [10]. This high-throughput technology is currently used with several platforms, including the Genome Analyzer/HiSeq/MiSeq (Illumina Solexa), the SOLiD System (Thermo Fisher Scientific), the Ion PGM/Ion Proton (Thermo Fisher Scientific), and the HeliScope Sequencer (Helicos BioSciences) [11, 12].

NGS can be used to detect both somatic and germline mutations in the cancer genome. The somatic genetic changes can be classified as either driver or passenger mutations. The former contribute to tumor development $[13,14]$, while the latter do not directly contribute to tumor development and may be the product of genomic instability within the tumor. Although SGS and PCR are routinely used to identify clinically relevant mutations and select the best treatment for patients, these techniques are insensitive to changes occurring at an allele frequency lower than $20 \%$, apart from real-time PCR, which could reach higher sensitivity $[15,16]$.

However, the more sensitive and cost-effective multiplex NGS testing platforms provide comprehensive genomic information, and thus allow for the implementation of targeted therapies and improved treatment decisions [17]. TP53 and PIK3CA are the most frequently mutated genes in breast cancer (BC), both being mutated in about onethird of all primary breast carcinomas $[18,19]$. In recent years, several studies identified the clinical relevance of PIK3CA mutations in terms of decreasing the benefits of anti-HER2 therapies and poly-chemotherapies in patients with PIK3CA mutations [20-22] . In the present study, we investigated the PIK3CA status of 186 primary BC patients from the Berlin area using targeted NGS and SGS. Recent studies have analyzed mutations in hot spots (i.e., exon 9 and 20) and only a few studies have analyzed mutations in other exons [23] Consequently, our aims were to evaluate the concordance between NGS and SGS for the most important hotspot regions in exon 9 and 20, to investigate additional hotspots outside of these exons using NGS, and to correlate the PIK3CA mutation status with the clinicopathological characteristics of the cohort.

\section{Methods}

\section{Patient cohort and histopathological evaluation}

Tissue samples were collected from 186 patients with a diagnosis of primary BC at the Department of Pathology, University Hospital Charité and the Breast Cancer Center at the DRK Klinikum Koepenick in Berlin, Germany. The median follow-up time was 38 months. Data on tumor histology and tumor grade were evaluated at the time of primary diagnosis and extracted from pathology reports. Tumors were graded according to the Bloom-Richardson grading system modified by Elston and Ellis [24]. HER2 status was determined by immunohistochemistry (IHC) using the Dako HercepTest kit (Dako, Carpinteria, CA, USA). Chromatic in situ hybridization (CISH) was also performed on samples with a HER2 score of $2+$. The estrogen receptor (ER) monoclonal antibody clone SP1 (NeoMarkers, Fremont, CA, USA) was used to identify the ER status, and the progesterone receptor (PR) status was determined with the PR monoclonal antibody PgR 636 (Dako, Wiesentheid, Germany). Only nuclear labeling was scored as positive. Negative ER and PR status was defined as positivity in $<1 \%$ of tumor cells according to ASCO/CAP guidelines [25]. HER2 negativity (HER2-) was defined as the absence of membranous staining or weak, discontinuous membranous staining. Cases with moderate membranous staining in $>10 \%$ of the tumor cells were examined by $\mathrm{CISH}$ according to ASCO/CAP guidelines [26]. A proliferation index was not available for all samples. Representative tumor samples containing at least $30 \%$ tumor cells were selected for molecular studies.

\section{Sample cohort and clinical parameters}

Median patient age at the time of diagnosis was 65 years, with a range of 34-95 years.

A total of 149 patients $(80.1 \%)$ had ductal carcinoma and $20(10.7 \%)$ had lobular carcinoma. Seventeen patients $(9.1 \%)$ had carcinoma of another histological type, such as mucinous ductal carcinoma with squamous differentiation, mixed-ductal and lobular carcinoma, medullary carcinoma, or invasive papillary adenocarcinoma. None of the patients received any medical treatment related to BC before surgery. After diagnosis, most (93\%) of the hormone receptor-positive $(\mathrm{HR}+)$ cases were administered hormonal therapy alone or in combination with other therapies according to relevant guidelines.

\section{Ethics approval}

Patients provided written informed consent for use of their biomaterial samples in biomarker studies. Consent was obtained using the standardized informed consent forms of the participating institutions. The project and consent process was approved by the ethics board of the Charité Hospital, Berlin (reference number EA1/139/05, last amendment 2013).

\section{DNA extraction, PCR, and PIK3CA semiconductor next- generation sequencing}

Briefly, 10 consecutive $10-\mu \mathrm{m}$ thick sections were prepared. The first section was stained with hematoxylin/ eosin and the tumor area was marked by a pathologist. The corresponding area was manually microdissected from each of the consecutive unstained sections and transferred to $180 \mu \mathrm{l}$ of lysis buffer (QIAamp DNA Mini 
Kit, Qiagen, Venlo, Niederlande) for $10 \mathrm{~min}$ at $95{ }^{\circ} \mathrm{C}$. Enzymatic lysis was carried out with $20 \mu \mathrm{l}$ of Proteinase $\mathrm{K}$ for $1 \mathrm{~h}$ at $56{ }^{\circ} \mathrm{C}$. Subsequent DNA preparation was performed according to the manufacturer's instructions, and the DNA was eluted in $80 \mu \mathrm{l}$ of elution buffer. Total nucleic acid concentrations were measured with a Qubit fluorometer HS DNA Assay (Life Technologies, Carlsbad, CA, USA) and a TaqMan RNase P Detection Reagents Kit (Life Technologies). Ten nanograms of genomic DNA were utilized for the library preparation. The final library was quantified using an Ion AmpliSeq Library Kit 2.0 (Life Technologies). The samples were 8-fold multiplexed and amplified on Ion Spheres Particles using the Ion OneTouch $^{\text {тs }} 200$ Template Kit v2 DL (Life Technologies). After library enrichment and quality control on a Qubit instrument (Ion Sphere Quality Control Kit, Life Technologies), the samples were sequenced using the Ion 318 chip v2 according to the standard protocol of the chip manufacturer.

A customized sequencing panel consisting of 154 amplicons from 48 genes was designed using the Ion AmpliSeq Designer to cover the most frequent somatic mutations found in BC. The panel included six amplicons located in PIK3CA exons 1, 4, 7, 9, 13, and 20. The genomic positions and primer sequences can be found in the well plate data sheet generated by the Ion AmpliSeq Designer (Additional file 1: Table S1). Only samples with at least $30 \%$ tumor cells within the dissected area were included in the study.

Base calling and alignment to the human genome (hg19) were executed with the Torrent Suite Software 4.0.3. The mean coverages (minimum - maximum) of the amplicons were 4128 bp (1315-22668 bp) for exon 1, 5237 bp (1962-25371 bp) for exon 4, 2044 bp (347-12066 bp) for exon 7, 3588 bp (278$20808 \mathrm{bp})$ for exon 9, $3742 \mathrm{bp}$ (1386-20063 bp) for exon 13, and 1552 bp (438-9901 bp) for exon 20. Variant calling was executed using the Torrent Variant Caller 4.2 and the low stringency somatic variant calling protocol. Only non-synonymous nucleotide exchanges were considered for the analysis of single nucleotide polymorphisms.

\section{Sanger sequencing primers and sequencing parameters} Primers were designed using the Primer Design Tool from NCBI. The primers were as follows:

exon 9 forward $5^{\prime}-$

GGGAAAAATATGACAAAGAAAGC-3',

exon 9 reverse 5'-GAGATCAGCCAAATTCAGTT-3', exon 20 part 1 forward $5^{\prime}$ -

CATTTGCTCCAAACTGACCA-3',

exon 20 part 1 reverse $5^{\prime}$ -

'TgTgCATCATTCATTTgTTTCA-3', exon 20 part 2 forward $5^{\prime}$ -

TTGATGACATTGCATACATTCG-3', and exon 20 part 2 reverse $5^{\prime}$ -

GGTCTTTGCCTGCTGAGAGT-3’.

The sequencing reactions were loaded on the 3730xl DNA Analyzer from Hitachi (Applied Biosystems). Sequence traces from tumor DNA samples were aligned to the genomic reference sequence and analyzed using SeqPilot software (Applied Biosystems).

\section{Statistical evaluation}

Statistical analyses were conducted using the SPSS 19 statistical software (SPSS Inc., Chicago, IL, USA) and the statistical language $\mathrm{R}$ (Foundation for Statistical Computing, Vienna, Austria). Significance of associations between PIK3CA status and age, ER/PR status, tumor stage, and histological grade were assessed using a Fisher's exact test, a chi-squared test, and a chi-squared test for trends. Overall survival was analyzed using the Kaplan-Meier method and the log-rank test. All tests were two-tailed, and results were considered significant when $p<0.05$. Barplots and beeswarm plots were produced using the $R$ package graphics and beeswarm [27, 28].

\section{Results}

\section{Prevalence of different types of PIK3CA mutations using} NGS

Using NGS to sequence the PIK3CA gene in each of the 186 tissue samples identified a total of 64 tumors with exon mutations (34.4\%), which agreed with the $36 \%$ PIK3CA mutation rate in $\mathrm{BC}$ reported by the Cancer Genome Atlas (TCGA) [18, 19]. As shown in Fig. 1, the mutations were distributed as follows: exon 20 (34 cases; $18.3 \%)$, exon 9 (19 cases; $10.2 \%)$, and other exons $(1,4$, 7 , or 13 ) (9 cases; $4.8 \%$ ). In very few samples (2 cases; $1.1 \%)$, we found mutations in two exons. The majority of mutations were base pair substitutions (60 cases, $93.8 \%$ ). Additionally, we detected deletions (4 cases, $6.3 \%)$. Sixty of the tumors (93.7 \%) had a single mutation in the PIK3CA gene, while three tumors had two mutations in the PIK3CA gene. The most frequent mutations were p.H1047R (31 cases, 48.4\%), p.E545K (11 cases, $17.2 \%$ ), and p.E542K (6 cases, 9.4 \%) (Table 1).

Three mutations, p.N347T, p.G451_D454del, and p.L456V, were not described in any of the studies reported in COSMIC (http://cancer.sanger.ac.uk/cancergenome/ projects/cosmic), TCGA $[18,19]$, or any other large genomic database [29, 30]. Thus, they are being described for the first time in our study. Additionally, a single nucleotide polymorphism (rs3729674) (NC_000003.11:g. $178917005 \mathrm{~A}>\mathrm{G}$ ) was found in 33 cases. Out of a total of 55 mutations in exons 9 and 20 that were detected using NGS, 49 were also detected by SGS. By resequencing 


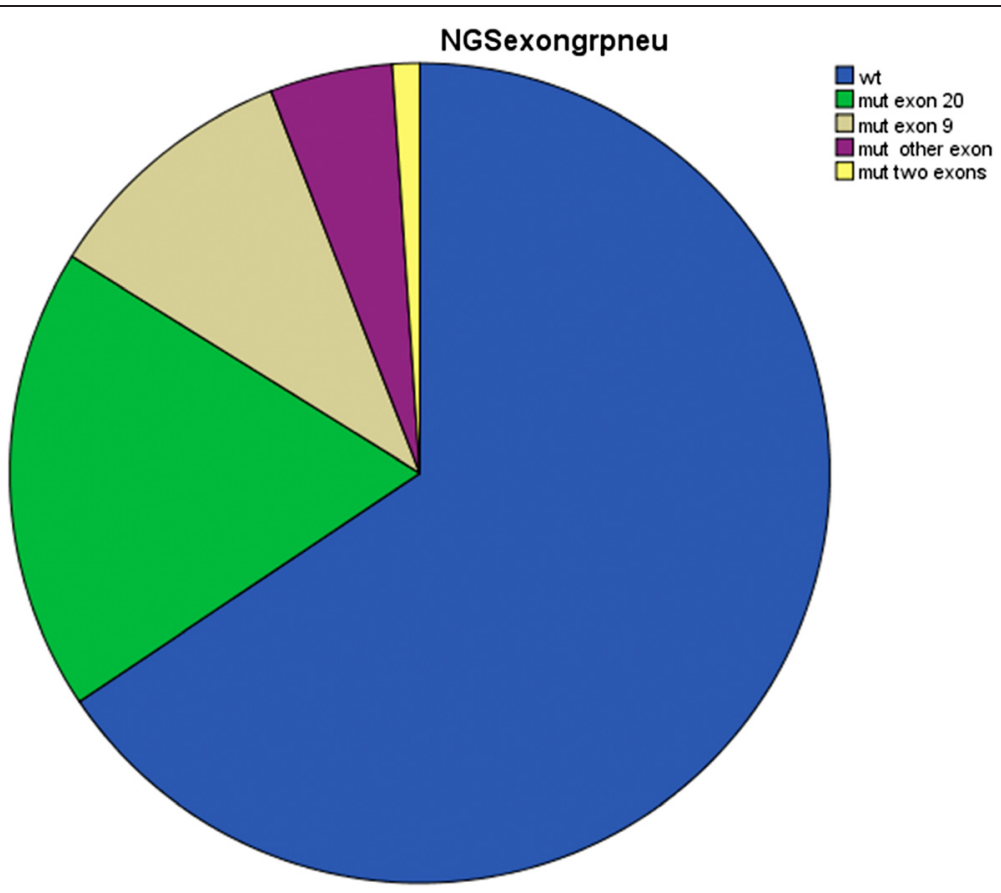

Fig. 1 PIK3CA mutations in breast cancer: frequency of mutations, including information on the affected exons

discrepant cases using SGS, we found an additional three cases with mutations with small peak heights that could be detected by analyzing the electropherograms manually. A comparison of the NGS and SGS results is shown in Fig. 2. The three mutations in exon 9 and exon 20 that were missed by SGS had low variant frequencies of $4 \%$ (twice) and $7 \%$.

\section{PIK3CA mutations and clinical characteristics}

PIK3CA mutations were analyzed for correlation with several clinicopathological parameters at the time of the diagnosis: age, tumor size, tumor grade, nodal status, HR status, HER2 expression, and histological subtype (Table 2). PIK3CA mutations were most frequently found in $\mathrm{HR}+$, HER2- $(p=0.002)$, and well-differentiated (G1; $p<0.001)$ cases (Fig. 3a and b). Furthermore, there was a statistically significant difference between cases with different nodal statuses; there were more cases with PIK3CA mutations in the N1 group $(p=0.042)$. We found no statistically significant correlation between mutation status and age, tumor size, or histological tumor type.

When we compared mutations in the other exons (1, 4,7 , and 13) with the clinicopathological parameters, we found a trend toward enrichment of the mutations in lower grade tumors, but this trend did not reach statistical significance $(p=0.104)$. There was no statistically significant difference between PIK3CA mutation status in these other exons and cases that were $\mathrm{HR}+, \mathrm{HR}-$, HER2+, or HER2-. Also, we could not detect differences in nodal status, tumor size, or age.

An overall survival analysis was performed on 184 patients with available follow-up data. In this group, no statistically significant association was found between long-term survival and the PIK3CA mutation status

Table 1 Hotspots of PIK3CA mutations in breast cancer

\begin{tabular}{lllll}
\hline Exon & Mutation & Number & Frequency (\%) & Total number \\
\hline 1 & R108del, R109del & 1,2 & $1.6,3.1$ & 3 \\
4 & N345K, N347T (new), D350N & $3,1,1$ & $4.7,1.6,1.6$ & 5 \\
7 & G451_D454del (new), p.L456V (new) & 1,1 & $1.6,1.6$ & 2 \\
9 & E542K, E545K, Q546K, Q546R & $6,11,1,1$ & $9.4,17.2$ & 19 \\
13 & E726K & 2 & 3,1 & 2 \\
20 & D1029H, N1044K, H1047L, H1047R, G1049R & $1,1,3,31,1$ & $1.6,1.6,48.4,4.7,1.6$ & 37
\end{tabular}

Using semiconductor NGS, we identified a total of 68 non-silent mutations in 186 breast cancer samples (34.4 \%). The most frequent aberrations were p.H1047R, p.E545K, and p.E542K 

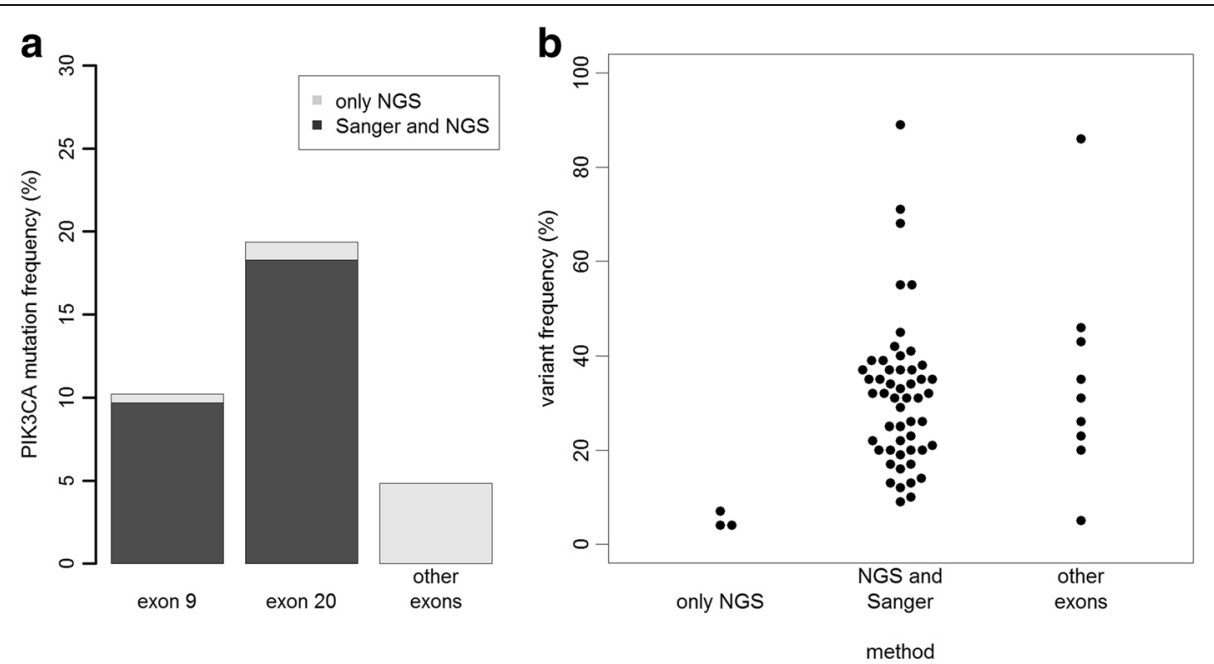

Fig. 2 Detection sensitivity of semiconductor NGS compared to Sanger sequencing. a Barplot showing the number of mutations detected by both Sanger sequencing and NGS and the mutations detected only by NGS. $\mathbf{b}$ Beeswarm plot showing the variant frequencies of the mutations detected only by NGS and those detected by both methods

(Fig. 4a). There was also no difference in overall survival related to the mutational status of different PIK3CA exons (Fig. 4b).

\section{Discussion}

The precise identification of genomic alterations is crucial for personalized cancer therapy. Molecular testing for mutations in cancer susceptibility genes is mainly performed using SGS of individual exons after PCR. The detection threshold of SGS requires an allele frequency of approximately $20 \%$ [31], but BC tissue is heterogeneous, consisting of tumor, stromal, and inflammatory cells, leading to a varying proportion of tumor cells ranging from 20-95\% [32]. Therefore, we speculated that a significant proportion of PIK3CA mutations are missed by SGS. There are studies available from other organ systems, including the lung, which report a higher detection rate of mutations using NGS rather than SGS [33, 34]. In general, the detection sensitivity of NGS reported in previous studies ranges from 94-99.9\% [35-39], which is above the sensitivity of Sanger Sequencing. Accordingly, the aim of the present study was to use NGS for the analysis of PIK3CA mutations to determine whether additional changes could be identified that might lead to better correlation of the clinicopathological characteristics of breast tumors with PIK3CA mutations. We also sought to evaluate the prognostic significance of PIK3CA mutation status as previously reported by several authors [40-44]. To this end, we compared the performance of NGS and SGS in the same cohort of patients, and identified PIK3CA mutations in $34.4 \%$ of breast tumors using NGS, which is in agreement with the mutation rate reported in TCGA [45].
Overall, we were able to report a good degree of concordance between the two sequencing methods. Only three mutations that were detected by NGS could not be found by SGS due to low variant frequencies below $10 \%$. Nevertheless, this finding indicates that NGS is more sensitive than SGS, particularly for the detection of low frequency mutations. This has also been reported in previous studies. Rohlin et al. [46] showed that Sangerbased sequencing techniques have problems picking out "minority" gene sequences (mutations below $15 \%$ ). Meanwhile, Walsch et al. performed a study on 300 high-risk $\mathrm{BC}$ families that screened for mutations in hotspots, and found previously undetected changes in 52 probands [47]. These results clearly support the use of targeted sequencing because it is more sensitive than SGS when it comes to identifying low frequency mutations. Our study, which is the first to compare NGS and SGS for sequencing the PIK3CA gene in $\mathrm{BC}$, adds support for this viewpoint. Additionally, the results of our study showed that $\sim 5 \%$ of the mutations were in other exons, and the best and most cost-effective method for detecting these mutations was to use parallel sequencing or targeted NGS. Due to the low mutation rate in these other exons $(1,4,7$, and 13) and a lack of statistical significance when correlated with clinicopathological data, we abstained from validating these mutations by SGS. The clinical relevance of PIK3CA mutations outside exon 9 and 20 should be further investigated in future studies.

The PIK3CA mutations detected by NGS in our study clustered in two previously reported "hotspot" regions in exons 9 and 20, with most of the mutations clustering in exon 20, which is in agreement with the SGS results reported in our previous study [48]. The consequences of 
Table 2 Correlation of PIK3CA mutation status with the clinicopathological characteristics of breast cancer

\begin{tabular}{|c|c|c|c|}
\hline Clinicopathological parameters & Mutated (\%) & Wild type (\%) & P \\
\hline All Tumor Cases & $64(34.4)$ & $122(65.6)$ & NS \\
\hline Histological Type & & & NS \\
\hline Ductal/Other Carcinoma & $53(32.5)$ & $110(67.5)$ & \\
\hline Lobular Carcinoma & $11(47.8)$ & $12(52.2)$ & \\
\hline Tumor Stage & & & NS \\
\hline $\mathrm{T} 1$ & $13(30.2)$ & $28(65.1)$ & \\
\hline T2 & $37(34.9)$ & $69(65.1)$ & \\
\hline T3 & $9(42.9)$ & $12(57.1)$ & \\
\hline T4 & $5(41.7)$ & $7(58.3)$ & \\
\hline Node Status & & & 0.042 \\
\hline NO & $25(27.8)$ & $65(72.2)$ & \\
\hline $\mathrm{N}+$ & $37(42.5)$ & $50(57.5)$ & \\
\hline Tumor Grade & & & $<0.001$ \\
\hline G1 & $16(80.0)$ & $4(20.0)$ & \\
\hline G2 & $35(36.5)$ & $61(63.5)$ & \\
\hline G3 & $13(18.8)$ & $56(81.2)$ & \\
\hline Hormone Receptor Status & & & 0.002 \\
\hline HR+ & $58(40.3)$ & $86(59.7)$ & \\
\hline HR- & $6(14.3)$ & $36(85.7)$ & \\
\hline HER2 Status & & & 0.032 \\
\hline HER2+ & $3(13.6)$ & $19(86.4)$ & \\
\hline HER2- & $61(37.2)$ & $103(62.8)$ & \\
\hline Age & & & NS \\
\hline$<50$ years & $6(26.1)$ & 17 (73.9) & \\
\hline$>50$ years & $58(35.6)$ & $105(64.4)$ & \\
\hline Molecular Type & & & 0.003 \\
\hline HR+/HER2- & $57(42.5)$ & 77 (57.5) & \\
\hline HR+/HER2+ & $1(10.0)$ & $9(90.0)$ & \\
\hline HR-/HER2+ & $2(16.7)$ & $10(83.3)$ & \\
\hline HR-/HER2- & $4(13.3)$ & $26(86.7)$ & \\
\hline
\end{tabular}

The mutation frequency, as determined by NGS, decreased with increasing tumor grade: $85 \%$ for G1, $37 \%$ for G2, and $20 \%$ for G3. PIK3CA mutations were more frequently detected (42\%) in $\mathrm{HR}+$ breast cancer than in HR- breast cancer (14\%). PIK3CA mutations were more frequently detected in HER2breast cancer (38\%) than in HER2+ breast cancer (14\%)

each mutation on the function and regulation of PIK3CA requires further consideration. The three novel mutation detected in our study are located in the $\mathrm{C} 2$ domain of the PIK3CA gene. The C2 is often involved in phospholipid membrane binding, consequently it is possible that these mutations lead to increased membrane binding, as extensively discussed in the study by Ikenoue at al. [49]. In the study by Gymnopoulos at al, the authors showed that the mutants in $\mathrm{C} 2$ domain increase basic positive surface charge of that domain and may therefore mediate improved recruitment of $\mathrm{p} 110 \alpha$ to the cell membrane, making lipid kinase activity independent of signals transmitted through the regulatory subunit, p85 [50]. One of these three mutations, p.L456V is predicted to be probably damaging with a score of 0.988 (sensitivity: 0.27 ; specificity: 0.99) when analyzed with polyPhen-2 prediction programe.

The goal of our previous study was to analyse only exon 9 and 20 mutations, hence we could not detect these three mutations in this study.

We also noted the presence of multiple mutations in four cases, which has not been reported previously. The significance of these double mutants is unknown, but it is possible that these tumors are multiclonal, and a second hit was required to provide a selective growth advantage if the first mutation was a less potent activator of the kinase.

We found that PIK3CA was most frequently mutated in cases that were HR+ and HER2-, which agrees with previously published data [51, 52]. We found significantly more PIK3CA mutations in G1 tumors suggesting that theses mutations occur early in BC development, which has also been shown in other studies [53]. Additionally, PIK3CA mutations were highly correlated with lymph node status $(\mathrm{N}+)$, which is one of the clinical markers associated with patient survival and response to therapy $[54,55]$. The finding that PIK3CA mutations are more commonly found in HR+ tumors may point to differences in pathogenesis and disease progression between $\mathrm{HR}+$ and HR- tumors. Furthermore, the correlation between PIK3CA mutations and lymph node metastasis suggests that activation of the PI3K/Akt pathway may increase the invasion of cancer cells into the lymph nodes. This is supported by the fact that PIP3 regulates cell mobility [56].

There is controversy regarding the prognostic significance of PIK3CA mutations. Cizkova et al. [57] described more favorable metastasis-free survival in patients with PIK3CA mutations, but Jensen et al. [22] and Baselga et al. [58] reported reduced survival rates and worse outcomes. The largest published study evaluated the PIK3CA genotype in 687 tumor samples from patients enrolled in a prospective phase III clinical trial. Those with PIK3CA mutations had a better prognosis for the first three years compared to those carrying wild type PIK3CA alleles, but this difference disappeared with a longer follow-up $[59,60]$. In our study, there was no significant association between PIK3CA mutational status and overall survival, indicating that an activated PIK3CA pathway alone is not a prognostic factor for $\mathrm{BC}$.

An interesting finding by $\mathrm{Fu}$ at al. showed that PIK3CA-activating mutations are associated with better outcomes in ER+ patients receiving endocrine therapy [61]. This agrees with the observation that PIK3CA mutations are more frequent in luminal A tumors compared 

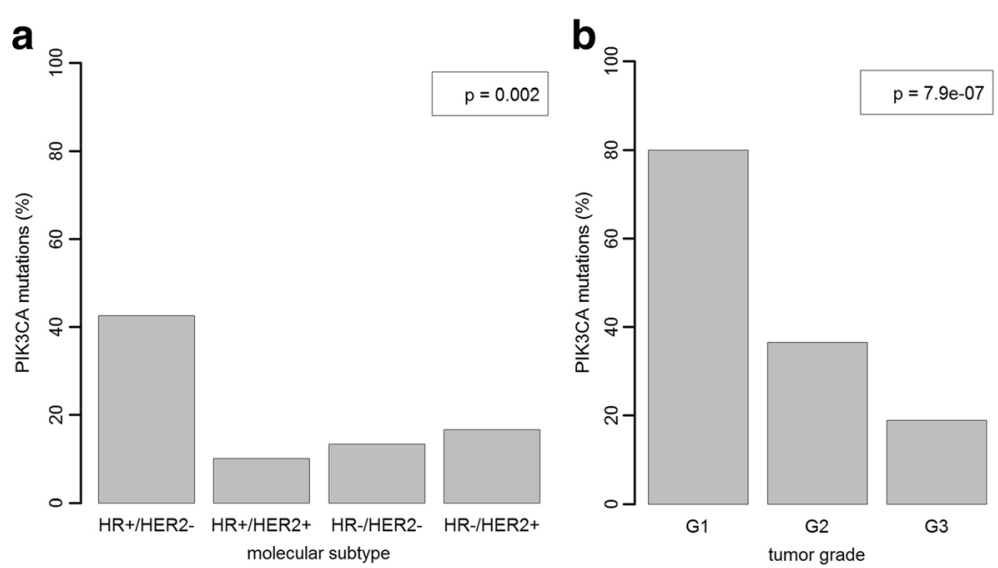

Fig. 3 Strong association of PIK3CA status with molecular subtype and tumor grade in breast cancer. a PIK3CA mutations were more frequent in HR+/HER2- breast cancer (40 \%) compared with the other subtypes (11\%-20\%). b The mutation frequency decreased with increasing tumor grade: $80 \%$ in G1, $36 \%$ in G2, and $19 \%$ in G3

with luminal B tumors (e.g., $45 \%$ vs. $29 \%$ in the TCGA cohort) [45]. In contrast, in HER2+ BC, several reports show that PIK3CA mutations predict adverse outcomes after treatment with trastuzumab [20, 21]. As such, the impact of PIK3CA mutations on the clinical outcome of $\mathrm{BC}$ seems to vary with the background of other genomic alterations such as HER2 status.

PIK3CA mutations also appear to have a significant interest in the prediction of response to targeted therapies, as many drugs specifically targeting PI3K or other effectors of the PI3K/AKT pathway are intended to be administered only to patients with tumor bearing a mutation of PIK3CA, which makes the somatic mutations detection more and more important [62].
In summary, our results show that NGS is more sensitive than SGS for detecting PIK3CA mutations in BC samples, and that PIK3CA mutations are significantly related to HR and HER2 expression status and tumor grade. Further studies are needed to systematically explore the functional relevance of PIK3CA mutations and the contribution of PIK3CA mediated activation of the downstream and upstream signaling pathways in breast tumor development and progression.

\section{Conclusions}

1. This is the first paper in which NGS and SGS were compared sequencing PIK3CA gene in breast cancer.
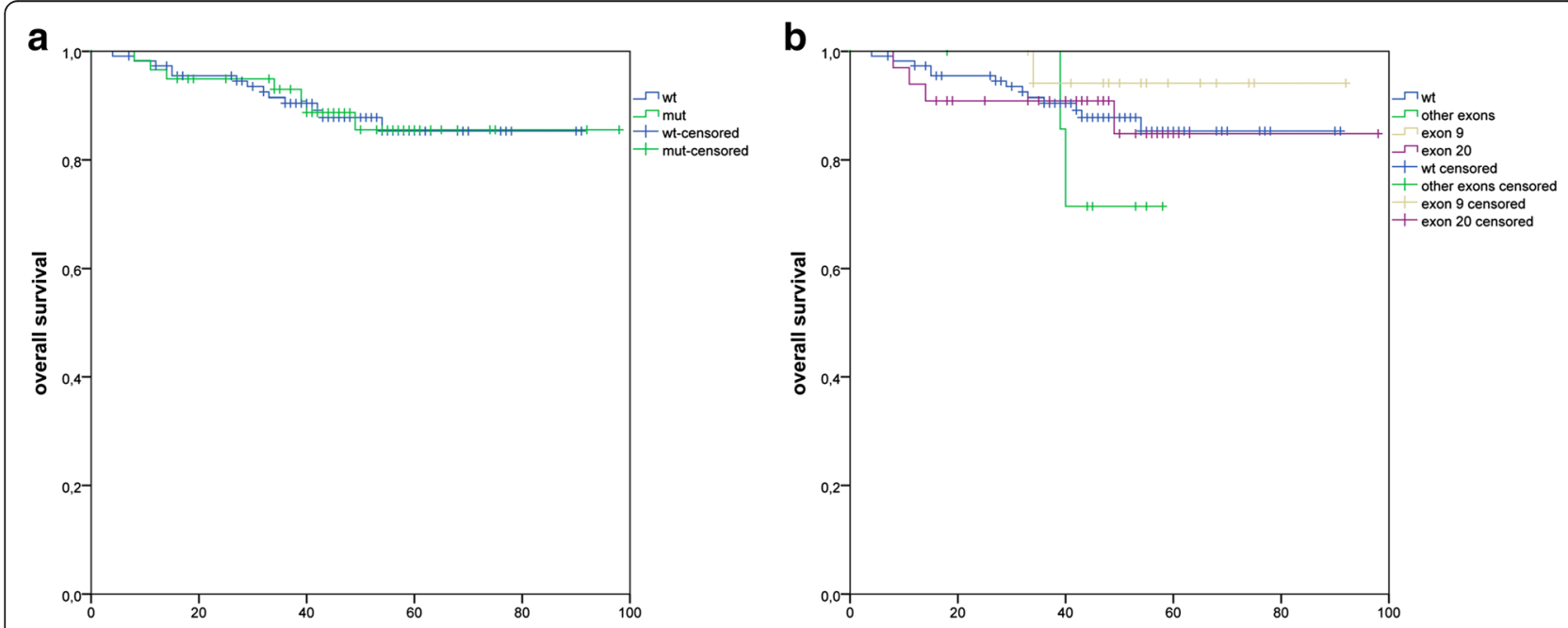

Fig. 4 Correlation of overall survival with the PIK3CA mutation status in breast cancer. a Kaplan-Meier analysis comparing patients with mutated PIK3CA (green line) and wild type PIK3CA (blue line) did not reveal a statistically significant difference in survival. b Kaplan-Meier analysis of the PIK3CA mutation status stratified for the affected exon did not reveal a statistically significant difference in survival, but there was a trend toward better survival for the cases with a mutation in exon 9. Exon 9 mutation $(p=0.39)$ vs. exon 20 mutation $(p=0.41)$ vs. other exon mutations ( $p=0.16)$ 
2. We found overall a good concordance between the two methods (98,4 \%), but better sensitivity of NGS when it comes to identifying low frequency mutations $(<10 \%)$.

3. PIK3CA mutation status in breast cancer correlated strongly with HR+ and HER2-, and N1 + .

\section{Additional file}

Additional file 1: Table S1. Genomic positions and the primer sequences. (XLS $45 \mathrm{~kb}$ )

\section{Competing interests}

The authors declare that they have no competing interests.

\section{Authors' contributions}

Each of the authors contributed to the manuscript. AR and JB wrote the manuscript and performed the analysis. JB performed the statistics. AL, DT, $\mathrm{MH}, \mathrm{CD}$ and $\mathrm{MD}$ corrected the manuscript. All authors are responsible for the overall content of the manuscript. All authors read and approved the final manuscript.

\section{Acknowledgments}

We would like to thank Ines Koch for the excellent technical assistance.

Received: 10 August 2015 Accepted: 12 November 2015

Published online: 18 November 2015

\section{References}

1. Chan M, Ji SM, Yeo ZH, Gan L, Yap E, Yap, YS et al. Development of a nextgeneration sequencing method for BRCA mutation screening a comparison between a high-throughput and a benchtop platform. J Mol Diagn. 2012; 14(6):602-12.

2. Chou LS, Liu CS-J, Boese B, Zhan X, Mao R. DNA Sequence Capture and Enrichment by Microarray Followed by Next-Generation Sequencing for Targeted Resequencing: Neurofibromatosis Type 1 Gene as a Model. Clin Chem. 2010;56(1):62-72.

3. De Leeneer, KHellemans J, De Schrivjer J, Baetens M, Poppe B., Van Criekinge W, et al. Massive parallel amplicon sequencing of the breast cancer genes BRCA1 and BRCA2: opportunities, challenges, and limitations. Hum Mutat. 2011;32(3):335-44.

4. Goossens D, Moens LN, Nelis E, Lenaerts AS, Glassee W, Kalbe A, et al. Simultaneous mutation and copy number variation (CNV) detection by multiplex PCR-based GS-FLX sequencing. Hum Mutat. 2009;30(3):472-6.

5. Hernan I, Borràs E, de Sousa Dias M, Gamundi MJ, Mañé B, Llort G, et al. Detection of genomic variations in BRCA1 and BRCA2 genes by long-range PCR and next-generation sequencing. J Mol Diagn. 2012;14(3):286-93.

6. Mamanova L, Coffey AJ, Scott CE, Kozarewa I, Turner EH, Kumar A, et al. Target-enrichment strategies for next-generation sequencing. Nat Methods. 2010;7(2):111-8

7. Ozcelik H, Shi X, Chang MC, Tram E, Vlasschaert M, Di Nicola N, et al. Longrange PCR and next-generation sequencing of BRCA1 and BRCA2 in breast cancer. J Mol Diagn. 2012;14(5):467-75.

8. Pritchard CC, Smith C, Salipante SJ, Lee MK, Thornton AM, Nord AS, et al. ColoSeq provides comprehensive lynch and polyposis syndrome mutational analysis using massively parallel sequencing. J Mol Diagn. 2012;14(4):357-66.

9. Walsh T, Lee MK, Casadei S, Thornton AM, Stray SM, Pennil C, et al. Detection of inherited mutations for breast and ovarian cancer using genomic capture and massively parallel sequencing. Proc Natl Acad Sci U S A. 2010;107(28):12629-33.

10. Walsh T, Casadei S, Lee MK, Pennil CC, Nord AS, Thornton AM, et al. Mutations in 12 genes for inherited ovarian, fallopian tube, and peritoneal carcinoma identified by massively parallel sequencing. Proc Natl Acad Sci U S A. 2011;108(44):18032-7.

11. Rothberg JM, Hinz W, Rearick TM, Schultz J, Mileski W, Davey M, et al. An integrated semiconductor device enabling non-optical genome sequencing. Nature. 2011;475(7356):348-52.
12. Voelkerding KV, Dames SA, Durtschi JD. Next-generation sequencing: from basic research to diagnostics. Clin Chem. 2009;55(4):641-58.

13. Hanahan D, Weinberg RA. The hallmarks of cancer. Cell. 2000;100(1):57-70.

14. Hanahan D, Weinberg RA. Hallmarks of cancer: the next generation. Cell. 2011;144(5):646-74.

15. MacConaill LE. Existing and emerging technologies for tumor genomic profiling. J Clin Oncol. 2013;31(15):1815-24.

16. Bustin SA. Absolute quantification of mRNA using real-time reverse transcription polymerase chain reaction assays. J Mol Endocrinol. 2000;25(2): 169-93.

17. Meyerson M, Gabriel S, Getz G. Advances in understanding cancer genomes through second-generation sequencing. Nat Rev Genet. 2010; 11(10):685-96

18. Forbes SA, Bindal N, Bamford S, Cole C, Kok CY, Beare D, et al. COSMIC: mining complete cancer genomes in the Catalogue of Somatic Mutations in Cancer. Nucleic Acids Res. 2011;39(Database issue):D945-50.

19. Robbins DE, Grüneberg A, Deus HF, Tanik MM, Almeida SJ. A self-updating road map of The Cancer Genome Atlas. Bioinformatics. 2013;29(10):1333-40.

20. Chandarlapaty S, Sakr RA, Giri D, Patil S, Heguy A, Morrow M, et al. Frequent mutational activation of the PI3K-AKT pathway in trastuzumab-resistant breast cancer. Clin Cancer Res. 2012;18(24):6784-91.

21. Cizkova M, Cizkova M, Dujaric ME, Lehmann-Che J, Scott V, Tembo O, et al. Outcome impact of PIK3CA mutations in HER2-positive breast cancer patients treated with trastuzumab. Br J Cancer. 2013;108(9):1807-9.

22. Jensen JD, Knoop A, Laenkholm AV, Grauslund M, Jensen MB, Santoni-Rugiu E, et al. PIK3CA mutations, PTEN, and pHER2 expression and impact on outcome in HER2-positive early-stage breast cancer patients treated with adjuvant chemotherapy and trastuzumab. Ann Oncol. 2012;23(8):2034-42.

23. Bai X, Zhang E, Ye H, Nandakumar V, Wang Z, Chen L, et al. PIK3CA and TP53 gene mutations in human breast cancer tumors frequently detected by ion torrent DNA sequencing. PLoS One. 2014;9(6):e99306.

24. Frierson Jr HF, Wolber RA, Berean KW, Franquemont DW, Gaffey MJ, Boyd JC, et al. Interobserver reproducibility of the Nottingham modification of the Bloom and Richardson histologic grading scheme for infiltrating ductal carcinoma. Am J Clin Pathol. 1995;103(2):195-8.

25. Hammond ME, Hayes DF, Dowsett M, Allred DC, Hagerty KL, Badve S, et al. American Society of Clinical Oncology/College of American Pathologists guideline recommendations for immunohistochemical testing of estrogen and progesterone receptors in breast cancer. Arch Pathol Lab Med. 2010; 134(6):907-22.

26. Wolff AC, Hammond ME, Hicks DG, Dowsett M, McShane LM, Allison KH, et al. Recommendations for human epidermal growth factor receptor 2 testing in breast cancer: American Society of Clinical Oncology/College of American Pathologists clinical practice guideline update. Arch Pathol Lab Med. 2014;138(2):241-56.

27. A.E. beeswarm: The bee swarm plot, an alternative to stripchart. R package version 0.1.6. 2013. http://CRAN.R-project.org/package=beeswarm.

28. R.C.T. R: A language and environment for statistical computing. R Foundation for Statistical Computing, Vienna, Austria. 2014. URL http://www.R-project.org/.

29. Cerami E, Gao J, Dogrusoz U, Gross BE, Sumer SO, Aksoy BA, et al. The cBio cancer genomics portal: an open platform for exploring multidimensional cancer genomics data. Cancer Discov. 2012;2(5):401-4.

30. Fokkema IF, Taschner PE, Schaafsma GC, Celli J, Laros JF, den Dunnen JT, et al. LOVD v. 2.0: the next generation in gene variant databases. Hum Mutat. 2011;32(5):557-63.

31. Kohlmann A, Klein HU, Weissmann S, Bresolin S, Chaplin T, Cuppens H, et al. The Interlaboratory RObustness of Next-generation sequencing (IRON) study: a deep sequencing investigation of TET2, CBL and KRAS mutations by an international consortium involving 10 laboratories. Leukemia. 2011; 25(12):1840-8

32. Cleator SJ, Powles TJ, Dexter T, Fulford L, Mackay A, Smith IE, et al. The effect of the stromal component of breast tumours on prediction of clinical outcome using gene expression microarray analysis. Breast Cancer Res. 2006;8(3):R32.

33. Buttitta F, Felicioni L, Del Grammastro M, Filice G, Di Lorito A, Malatesta S, et al. Effective assessment of egfr mutation status in bronchoalveolar lavage and pleural fluids by next-generation sequencing. Clin Cancer Res. 2013; 19(3):691-8.

34. Moskalev EA, Stöhr R, Rieker R, Hebele S, Fuchs F, Sirbu H, et al. Increased detection rates of EGFR and KRAS mutations in NSCLC specimens with low tumour cell content by 454 deep sequencing. Virchows Arch. 2013;462(4): 409-19. 
35. Chin ELH, C da Silva, M Hegde. Assessment of clinical analytical sensitivity and specificity of next-generation sequencing for detection of simple and complex mutations. BMC Genet. 2013;14:14-6

36. Guan YF, Hu H, Peng Y, Gong Y, Yi Y, Shao L, et al. Detection of inherited mutations for hereditary cancer using target enrichment and next generation sequencing. Fam Cancer. 2015;14(1):9-18.

37. Hadd AG, Houghton J, Choudhary A, Sah S, Chen L, Marko AC, et al. Targeted, high-depth, next-generation sequencing of cancer genes in formalin-fixed, paraffin-embedded and fine-needle aspiration tumor specimens. J Mol Diagn. 2013;15(2):234-47.

38. Lin MT, Mosier SI, Thiess M, Beierel KF, Debeljak M, Tseng LH, et al. Clinical Validation of KRAS, BRAF, and EGFR mutation detection using nextgeneration sequencing. Am J Clin Pathol. 2014;141(6):856-66.

39. Nijman IJ, van Montfrans JM, Hoogstraat M, Boes ML, van de Corput L, Renne ED, et al. Targeted next-generation sequencing: a novel diagnostic tool for primary immunodeficiencies. J Allergy Clin Immunol. 2014;133(2):529-34.

40. Barbareschi M, Buttitta F, Felicioni L, Cotrupi S, Barassi F, Del Grammastro M, et al. Different prognostic roles of mutations in the helical and kinase domains of the PIK3CA gene in breast carcinomas. Clin Cancer Res. 2007; 13(20):6064-9.

41. Lai YL, Mau BL, Cheng WH, Chen HM, Chiu HH, Tzen CY. et al. PIK3CA exon 20 mutation is independently associated with a poor prognosis in breast cancer patients. Ann Surg Oncol. 2008;15(4):1064-9.

42. Li SY, Rong M, Grieu F, lacopetta B. PIK3CA mutations in breast cancer are associated with poor outcome. Breast Cancer Res Treat. 2006;96(1):91-5.

43. Mangone FR, Bobrovnitchaia IG, Salaorni S, Manuli E, Nagai MA.PIK3CA exon 20 mutations are associated with poor prognosis in breast cancer patients. Clinics (Sao Paulo). 2012;67(11):1285-90

44. Saal LH, Holm K, Maurer M, Memeo L, Su T, Wang X, et al. PIK3CA mutations correlate with hormone receptors, node metastasis, and ERBB2, and are mutually exclusive with PTEN loss in human breast carcinoma. Cancer Res. 2005;65(7):2554-9.

45. Koboldt DC, Fulton RS, McLellan, MD, Schmidt H, Kalicki-Veizer J, McMichael, JF, et al. Comprehensive molecular portraits of human breast tumours. Nature. 2012;490(7418):61-70.

46. Rohlin A, Wernersson J, Engwall Y, Wiklund L, Bjoerk J, Nordling M, et al. Parallel sequencing used in detection of mosaic mutations: comparison with four diagnostic DNA screening techniques. Hum Mutat. 2009;30(6): 1012-20.

47. Walsh T, Casadei S, Coats KH, Swisher E, Stray SM, Higgins J, et al. Spectrum of mutations in BRCA1, BRCA2, CHEK2, and TP53 in families at high risk of breast cancer. JAMA. 2006;295(12):1379-88.

48. Arsenic R, Lehmann A, Budczies J, Koch I, Prinzler J, Kleine-Tebbe A, et al. Analysis of PIK3CA mutations in breast cancer subtypes. Appl Immunohistochem Mol Morphol. 2014;22(1):50-6.

49. Ikenoue T, Kanai F, Hikiba Y, Obata T, Tanaka Y, Imamura J, et al. Functional analysis of PIK3CA gene mutations in human colorectal cancer. Cancer Res. 2005;65(11):4562-7.

50. Gymnopoulos M, Elsliger MA, Vogt PK. Rare cancer-specific mutations in PIK3CA show gain of function. Proc Natl Acad Sci U S A. 2007;104(13):5569-74.

51. Boyault S, Drouet $Y$, Navarro C, Bachelot T, Lasset C, Treilleux I, et al. Mutational characterization of individual breast tumors: TP53 and PI3K pathway genes are frequently and distinctively mutated in different subtypes. Breast Cancer Res Treat. 2012;132(1):29-39.

52. Kalinsky K, Jacks LM, Heguy A, Patil S, Drobnjak M, Bhanot UK, et al. PIK3CA mutation associates with improved outcome in breast cancer. Clin Cancer Res. 2009;15(16):5049-59.

53. Dunlap J, Le C, Shukla A, Patterson J, Presnell A, Heinrich MC, et al. Phosphatidylinositol-3-kinase and AKT1 mutations occur early in breast carcinoma. Breast Cancer Res Treat. 2010;120(2):409-18.

54. Hutter RV. Pathological parameters useful in predicting prognosis for patients with breast cancer. Monogr Pathol. 1984;25:175-85.

55. McGuire WL, Clark, GM, Dressler LG and Owens, MA. Role of steroid hormone receptors as prognostic factors in primary breast cancer. $\mathrm{NCl}$ Monogr. 1986;1:19-23.

56. Huang $Y E$, lijima $M$, Parent $C A$, Funamoto $S$, Firtel RA,Devreotes $P$, et al. Receptor-mediated regulation of PI3Ks confines PI(3,4,5)P3 to the leading edge of chemotaxing cells. Mol Biol Cell. 2003;14(5):1913-22.

57. Cizkova M, Susini A, Vacher S, Cizeron-Clairac G, Andrieu C, Driouch K, et al. PIK3CA mutation impact on survival in breast cancer patients and in ER alpha, PR and ERBB2-based subgroups. Breast Cancer Res. 2012;14(1):R28.
58. Baselga J, Cortés J, Im SA, Clark E, Ross G, Kiermaier A, et al. Biomarker analyses in CLEOPATRA: a phase III, placebo-controlled study of pertuzumab in human epidermal growth factor receptor 2-positive, first-line metastatic breast cancer. J Clin Oncol. 2014;32(33):3753-61.

59. Joensuu H, Kellokumpu-Lehtinen PL, Bono P, Alanko T, Kataja V, Asola R, et al. Adjuvant docetaxel or vinorelbine with or without trastuzumab for breast cancer. N Engl J Med. 2006;354(8):809-20.

60. Loi S, Michiels S, Lambrechts D, Fumagalli D, Claes B, Kellokumpu-Lehtinen $\mathrm{PL}$, et al. Somatic mutation profiling and associations with prognosis and trastuzumab benefit in early breast cancer. J Natl Cancer Inst. 2013;105(13): 960-7.

61. Fu X, Osborne CK, Schiff R. Biology and therapeutic potential of PI3K signaling in ER+/HER2-negative breast cancer. Breast. 2013;22 Suppl 2:S12-8.

62. Harle A, Lion M, Lozano N, Merlin JL. Clinical, diagnostic significance and theranostic interest of PIK3CA gene mutations in breast cancer. Bull Cancer. 2013;100(10):947-54.

\section{Submit your next manuscript to BioMed Central and take full advantage of:}

- Convenient online submission

- Thorough peer review

- No space constraints or color figure charges

- Immediate publication on acceptance

- Inclusion in PubMed, CAS, Scopus and Google Scholar

- Research which is freely available for redistribution 